\title{
Open Source
}

\section{Hintergrund}

Geistesschöpfungen einer Person wie literarische oder musische Werke, aber auch wissenschaftliche und journalistische Texte sowie seit 1993 auch Software unterliegen in Deutschland dem Urheberrecht. Es schützt als Ausfluss des allgemeinen Persönlichkeitsrechts das geistige Eigentum des Herstellers; auf diesen Schutz kann der Urheber des Werkes (wenigstens in Deutschland) nicht einmal selbst verzichten.

Mit der Verbreitung von Home- und Personal Computern zu Beginn der 80er Jahre des 20. Jahrhunderts entstand eine Vielzahl kleiner und größerer Computerprogramme, die von ihren Schöpfern unentgeltlich verbreitet wurden. Der dafür ursprünglich verwendete Begriff der „Public Domain“Software war irreführend, denn „Gemeinfreiheit" impliziert das Fehlen eines Urheberschutzes. Inzwischen hat sich für solcherart unentgeltlich verbreitete Software der zutreffendere Begriff „Freeware“ etabliert. Abweichend davon bezeichnet „Shareware" Programme, die in einer (zeitlich oder funktional) eingeschränkten Version unentgeltlich verbreitet werden; bei Erwerb einer Nutzungslizenz erhält sie den vollständigen Funktionsumfang.

„Freeware" und „Shareware“ werden in der Regel als ausführbare Programme verbreitet, d.h. der Nutzer erhält keinen Quellcode und kann das Programm auch nicht ohne Weiteres verändern. Nachteil: Das Programm kann nur von dem oder den Urhebern selbst weiter entwickelt werden.

Diesem Nachteil wollte Richard Stallman, Gründer des GNU1-Projekts zur Entwicklung eines „freien Unix-artigen Betriebssystems“ (1984), durch die Entwicklung ,freier“ Lizenzbedingungen abhelfen, um möglichst viele, unabhängige Programmierer für die Mitwirkung am GNU-Projekt gewinnen zu können. 1985 gründete Stallman die gemeinnützige „Free Software Foundation“ (FSF), die das GNU-Projekt seither trägt. Für die Offenlegung des Programm-Codes fehlte allerdings noch eine urheberrechtliche Lösung - wie sollte der Umgang mit dem offenen Code geregelt werden?

GNU $=$ „GNU is not Unix“

\section{GPL - GNU General Public License}

Zusammen mit Jerry Cohen entwickelte Stallman aus Einzelregelungen für die Projekte GNU Emacs, GNU Compiler Collection und GNU Debugger eine vereinheitlichte GNU General Public Licence (GPL) ${ }^{2}$, deren erste Version 1989 veröffentlicht wurde.

Im Kern umfasst die GPL die folgenden vier elementaren „Freiheiten“:

1. Das unter GPL verbreitete Programm darf für jeden Zweck genutzt werden.

2. Kopien des Programms dürfen kostenlos, aber nur zusammen mit dem Quellcode verteilt werden.

3. Die Funktionsweise des Programms darf analysiert und an die eigenen Bedürfnisse angepasst werden.

4. Auch dürfen angepasste Versionen des Programms verbreitet werden; dabei muss dem Empfänger des Programms allerdings der gesamte Quellcode verfügbar gemacht werden.

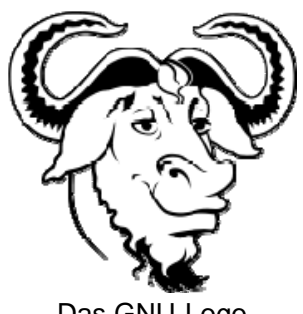

Das GNU-Logo

GPL ist heute die weltweit verbreitetste Software-Lizenz. Der Index der FSF umfasst mehr als 4.700 unter der GPL entwickelte Programme, darunter Linux und das PGP-kompatible Verschlüsselungspaket GnuPG (GPG).

Eine Erweiterung und Flexibilisierung der GPL (Version 3) ist derzeit in Abstimmung; sie wird voraussichtlich Anfang 2007 veröffentlicht. Sie soll internationale Regelungen des Urheberrechts angemessener berücksichtigen.

\section{Open Source Definition}

Der Begriff „Open Source“ ist wesentlich jünger und geht auf Bruce Perens zurück,

\footnotetext{
${ }^{2}$ http://www.gnu.de/gpl-ger.html
}

Entwickler von Debian GNU/Linux. Er gründete 1998 die „Open Source Initiative“ $(\mathrm{OSI})^{3}$ in Abgrenzung zu Richard Stallmans FSF. Die von der OSI entwickelte „Open Source Definition“ (OSD), hervorgegangen aus dem „Debian Social Contract“, ist ein Rahmenwerk für „Open Source Lizenzen“. Es legt unter anderem fest, dass

1. die Lizenz keine Personen, Gruppen oder Einsatzgebiete des Programms diskriminieren darf,

2. bei der Weitergabe an Dritte die Lizenz gelten soll, ohne dass Urheber und Lizenznehmer einen Vertrag unterschreiben, und

3. das Recht zur Weitergabe nicht an eine bestimmte Distribution gebunden werden darf.

Lizenzen, die die OSD-Bedingungen erfüllen, dürfen sich - nach Anerkennung durch die OSI - mit dem geschützten Trademark „OSI Certified Open Source Software“ schmücken. $\mathrm{Zu}$ den anerkannten Lizenzen zählen u.a. die Mozilla Public License, die Apache License und die New BDS License.

\section{Klarstellungen}

„Open Source“ bezieht sich - anders als „Freeware" - begrifflich zunächst allein auf die Frage der unbeschränkten Offenlegung des Programm-Quellcodes. Ob Open Source kostenlos oder nur gegen eine Lizenzgebühr genutzt werden darf, ist von dieser Frage unabhängig - Open Source ist also keineswegs automatisch „Gratissoftware“. Geringe Kosten sind also kein Argument für „Open Source“, sondern allein für „Freeware", auch wenn viele Open Source-Programme tatsächlich zugleich „Freeware“ sind.

Umgekehrt bleibt bei kommerzieller Software der Quellcode dem Kunden nicht zwangsläufig verborgen: Einige Hersteller legen den Code ihren Kunden nach Abschluss einer Vertraulichkeitsvereinbarung offen. Die Weiterentwicklung des Codes ist jedoch auf den Hersteller beschränkt, die Offenlegung auf tatsächliche Käufer.

\footnotetext{
${ }^{3}$ http://www.opensource.org/
} 